Chapter 8

\title{
MASK PATTERN TRANSFERRED TRANSIENT GRATING TECHNIQUE FOR MOLECULAR-DYNAMICS STUDY IN SOLUTIONS
}

\subsection{Introduction}

The transient grating (TG) technique $[1,2]$, which is one of the third-order nonlinear spectroscopies, has been a powerful tool to monitor several photonics and photochemical processes in solutions since its outset. For a brief history, solutions were photoexcited by an optical interference pattern generated by two crossing pump beams. As parameters, several properties of a solution were spatially modulated according to the interference pattern and were detected through the diffraction of the probe beam. The time-varying profiles of the diffracted signal indicate the many processes in solution, such as electron transfer, molecular dynamics, heat dynamics, ultrasonics, volume/structure change, clustering/aggregation, and chemical reaction [3-8]. The optical heterodyne detected-TG (OHD-TG) technique [9-16] was also developed later to improve sensitivity. The reference beam, which has the same direction, polarization, and wavelength as the signal beam, was superimposed on the signal beam to amplify the signal intensity. The experimental setups of the OHD-TG were very difficult because the probe and reference beams need to be aligned and tuned within submicron meter scale to meet the phase-matching condition. Very recently, Katayama et al. developed a lens-free OHD-TG technique $[17,18]$ by using a transmission grating structure in a $3 \mathrm{~mm}$ glass. This technique was easier than the traditional OHD-TG technique. In this chapter, we describe a more convenient and easy 
well aligned technique; the mask pattern transferred-TG (MPT-TG) technique-by using a metal film grating fabricated in our laboratory.

\subsection{Experimental setup}

Figure 8.1a shows the scanning electron microscope (SEM) image of the smallest metal grating film (400 $\mathrm{nm}$ metal width and $1 \mu \mathrm{m}$ period). Chromium layer (100 nm) was created by vacuum evaporation on the quartz substrate. A fine grade resist was deposited on a $\mathrm{Cr}$ layer by spin coating. Nano or micrometer scaled patterns are written by the lithography

(a)

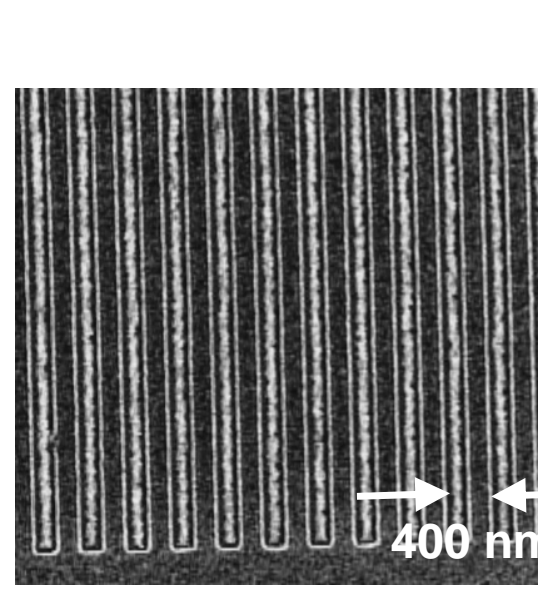

(b)

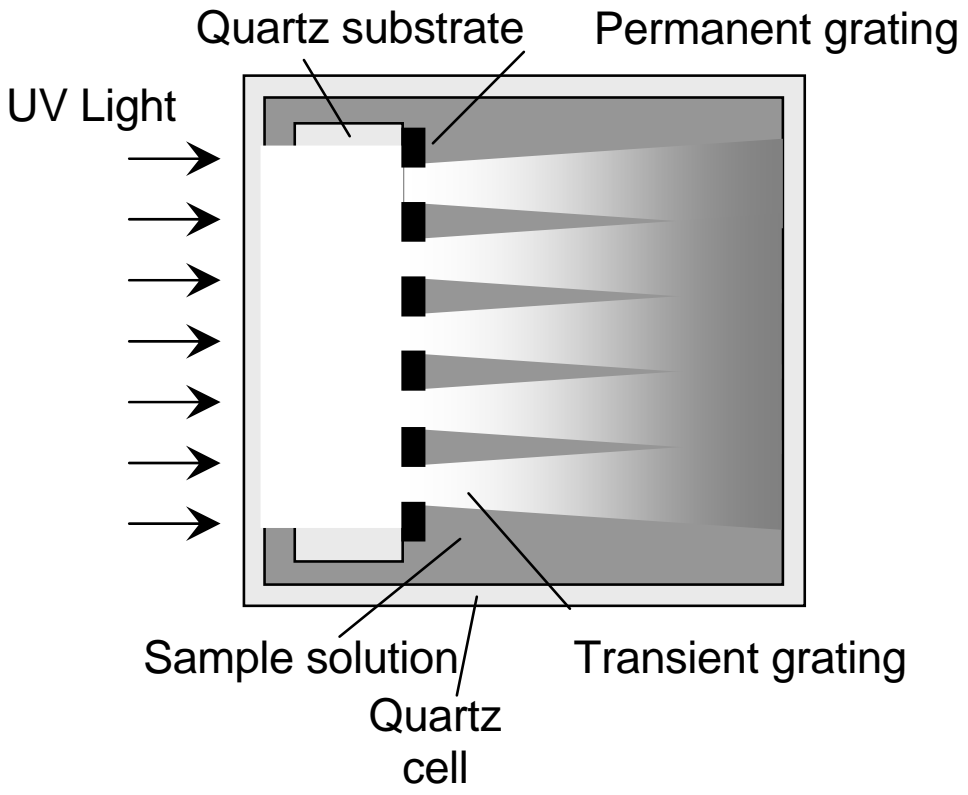

Figure 8.1 (a) Scanning electron microscope (SEM) image of the smallest fabricated nano metal grating. (b) Schematic diagram of the pattern transfer from the metal grating film to the solution

of an electron beam or a direct writing laser. Finally, metal grating structures are engraved by chemical etching. The principle of the MPT-TG is depicted in Figure 8.1b. A finished metal grating film is suspended into the sample solution and the excitation UV pump beam 
$\left(I_{\mathrm{e}}\right)$ is incident at the grating in solution. Thus the grating pattern is transferred from the metal film to the sample solution under near-field condition (Fresnel diffraction). This is similar to traditional photo-lithography but, in this case, the sample material is liquid solution. The solute molecules were excited by this special transient grating (TG) structure. A part of the probe beam $\left(I_{\mathrm{p}}\right)$ irradiated on the grating in the solution is diffracted under the far-field condition (Fraunhoffer diffraction) and was detected by the photodetector.

The experimental setup of the MGT-TG is schematically shown in Figure 8.2. A

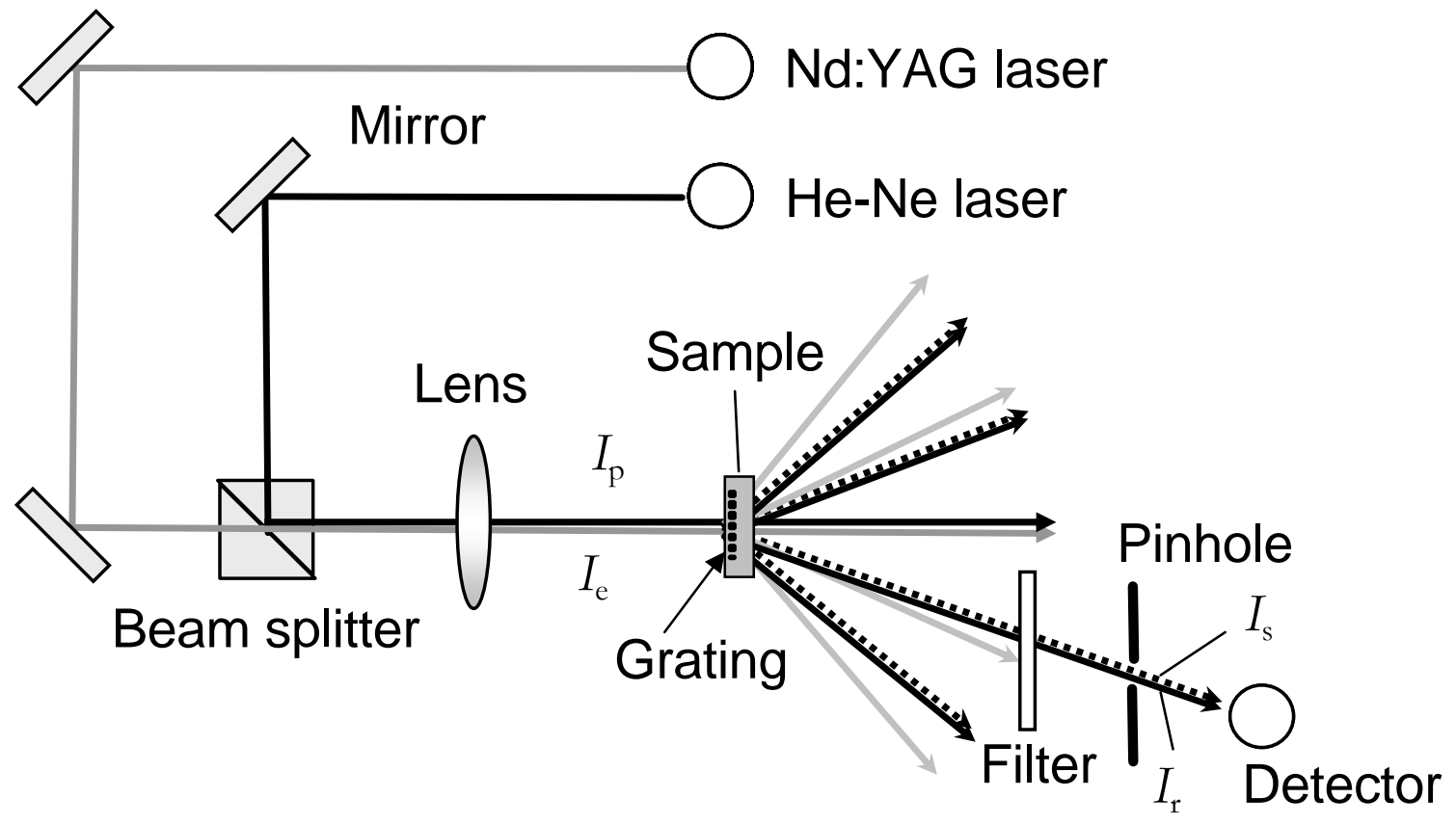

Figure 8.2 Experimental setup of the MPT-TG technique with metal grating and the optical configurations of irradiated pump $\left(I_{e}\right)$ and probe $\left(I_{p}\right)$ beams and diffracted reference $\left(I_{r}\right)$ and signal $\left(I_{s}\right)$ beams

frequency-tripled Nd:YAG laser $\left(\lambda_{\mathrm{e}}=366 \mathrm{~nm}, I_{\mathrm{e}}=0.3 \mathrm{~mJ} /\right.$ pulse $)$ was used as a pump beam. The pulse width and repetition rate are $10 \mathrm{~ns}$ and $3 \mathrm{~Hz}$, respectively. A cw-He-Ne laser $\left(\lambda_{\mathrm{p}}\right.$ $=633 \mathrm{~nm}, I_{\mathrm{p}}=0.05 \mathrm{~mW}$ ) is used as a probe beam. Both beams are focused by a lens on the 
sample solution in a quartz cell (10 mm spacing). A limited diffraction beam passes through a pinhole and a glass filter and is registered with an InGaAs photodetector. Two types of signals, diffracted by permanent metal grating $\left(I_{\mathrm{r}}\right)$ and transient grating $\left(I_{\mathrm{S}}\right)$, are simultaneously detectable. The permanent grating signal plays the role of the reference beam of the OHD-TG setup and amplifies the transient grating signal. Both diffracted signals pass the color filter and pinhole to be separated from the pump beam and detected onto the photodetector.

Sample solution was nitrobenzene in 2-propanol (5 volume \%). The excited energy of nitrobenzene is immediately converted to molecular vibration, translation, and finally heat energy. Such non-radiative relaxation processes of nitrobenzene are completed within a very short time-scale (a few hundred picoseconds) [19]. Therefore, nitrobenzene has been used often as the standard solution of the typical heat source (molecular heater) for photothermal measurement.

\subsection{Results and discussion}

Figure 8.3 shows the semi-log plot of the time-dependent diffraction signals with (a) micron-meter scaled and (b) nano-meter scaled etch width metal grating, respectively. We found the exponential decay component $\left(I_{\mathrm{s}}\right)$ superimposed on the large non-decayed offset component $\left(I_{\mathrm{r}}\right)$. The former is due to the transient grating while the latter is due to the permanent grating, respectively. According to the theory, detected total signal intensity $\left(I_{\text {total }}\right)$ can be described by $[17,18]$

$$
I_{\text {total }}(t)=I_{r}+2 a\left[\chi^{(3)} \cdot(t) \cos \Delta \phi+\chi^{(3)} "(t) \sin \Delta \phi\right] I_{e} I_{p}+\left|\chi^{(3)}(t)\right|^{2} I_{e}^{2} I_{p}
$$



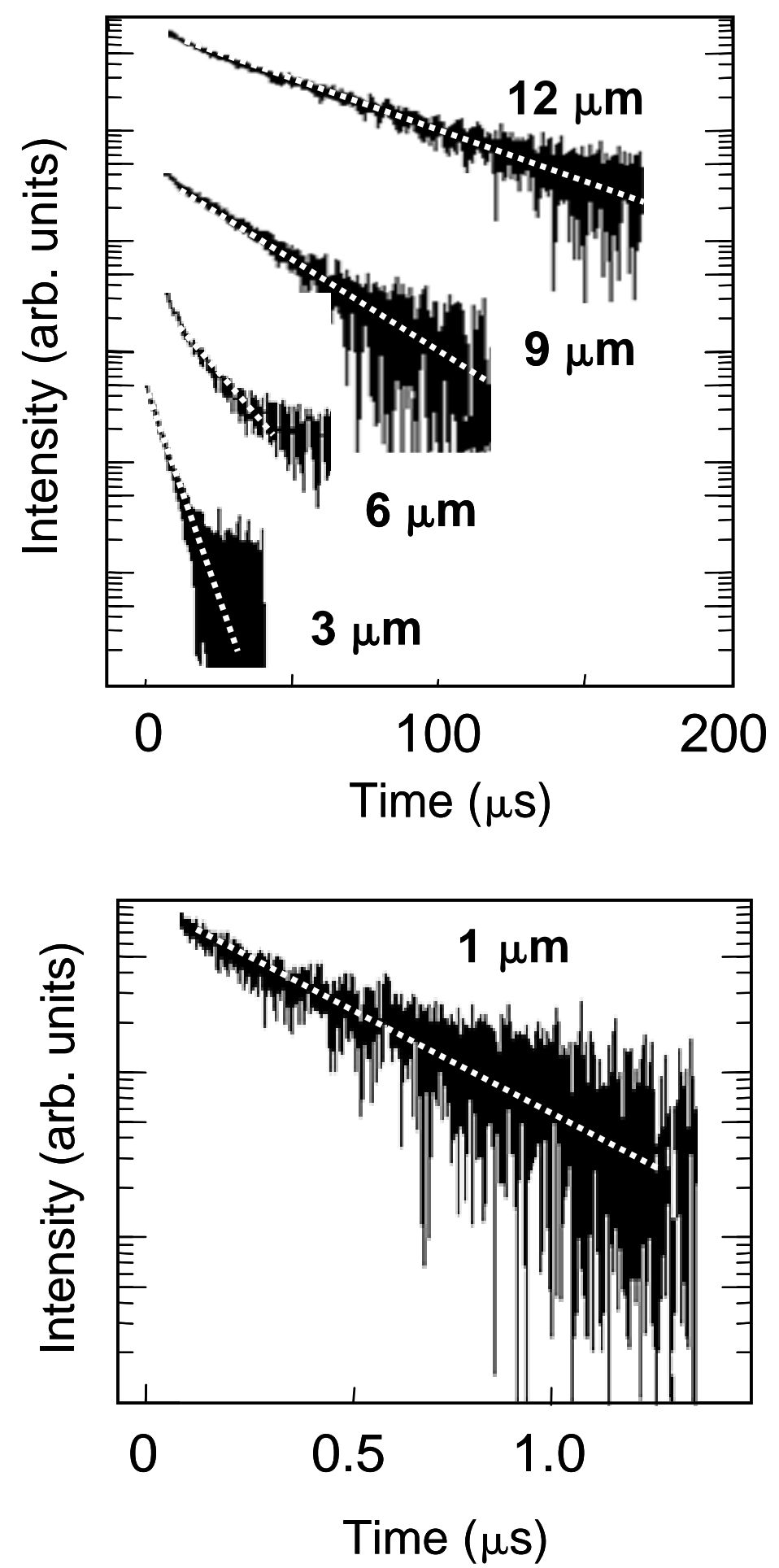

Figure 8.3 Time profile of the diffracted signals with metal grating of $12,9,6$, and $3 \mathrm{\mu m}$ periods (a) and $1 \mathrm{\mu m}$ period (b). Dashed lines were fitted by the exponential functions. 
where $\chi^{(3) '}(t)$ and $\chi^{(3), '}(t)$ are the real part and imaginary part, respectively, of the thirdorder nonlinear electrical susceptibility $\chi^{(3)}(t)$, and $a$ is a real constant. The third term indicates the TG signal with usual homodyne detection and is negligible because involved $\chi^{(3)}$ in solution should be very small. Thus, only the second term of equation (8.1) indicates $I_{\mathrm{s}}$. In this case, $\chi^{(3),}(t)$ and $\chi^{(3), '(t)}$ are equal to the refractive index change $\delta n(t)$ and the absorbance change $\delta k(t)=0$ at $633 \mathrm{~nm}$ induced by the transient grating, respectively. $\Delta \phi$ is the phase difference between $I_{\mathrm{r}}$ and $I_{\mathrm{p}}$. In the OHD-TG techniques, unstable $\Delta \phi$ has been the main difficulty of the experimental setup. However, in our setup, $\Delta \phi$ is decided only by the structure and thickness of the metal grating. Therefore, the phase stability is excellent without the tuning of beam lines in this setup. Thus, $\sin \Delta \phi$ in equation (8.1) should be a constant, and $I_{\mathrm{S}}$ should be proportional to $\delta n(t)$.

The spatial modulation of the optical intensity $\left(\delta I_{\mathrm{e}}\right)$ induced the spatial modulation of the population of the molecular excited states $(\delta P)$, temperature $(\delta T)$, and density of solvent $(\delta \rho)$. Namely, $\delta n$ should be attributed to $\delta T$ and $\delta \rho$ in this time scale. Temperature rising increases $\delta \rho$, which decreases $\delta n$. Therefore, spatial distribution of temperature (thermal grating) was created, and the signal decay shows the thermal diffusion processes in solution. By solving the Fourier's diffusion equation, the time profile of $\delta n(t)$ is given by [20]

$\delta \hat{n}(t)=\left[\left(\frac{\partial n}{\partial \rho}\right)_{T}\left(\frac{\partial \rho}{\partial T}\right)+\left(\frac{\partial n}{\partial T}\right)_{\rho}\right] \frac{Q}{\rho C_{p}} \delta I_{e}[C] \exp \left(-D_{t h} q^{2} t\right)$

where, $Q, C_{\mathrm{p}}$, and $[C]$ are the heat energy released from unit molecules, specific heat capacity, and solute molecular concentration. $\delta \hat{n}(t)$ is the spatial Fourier component of 


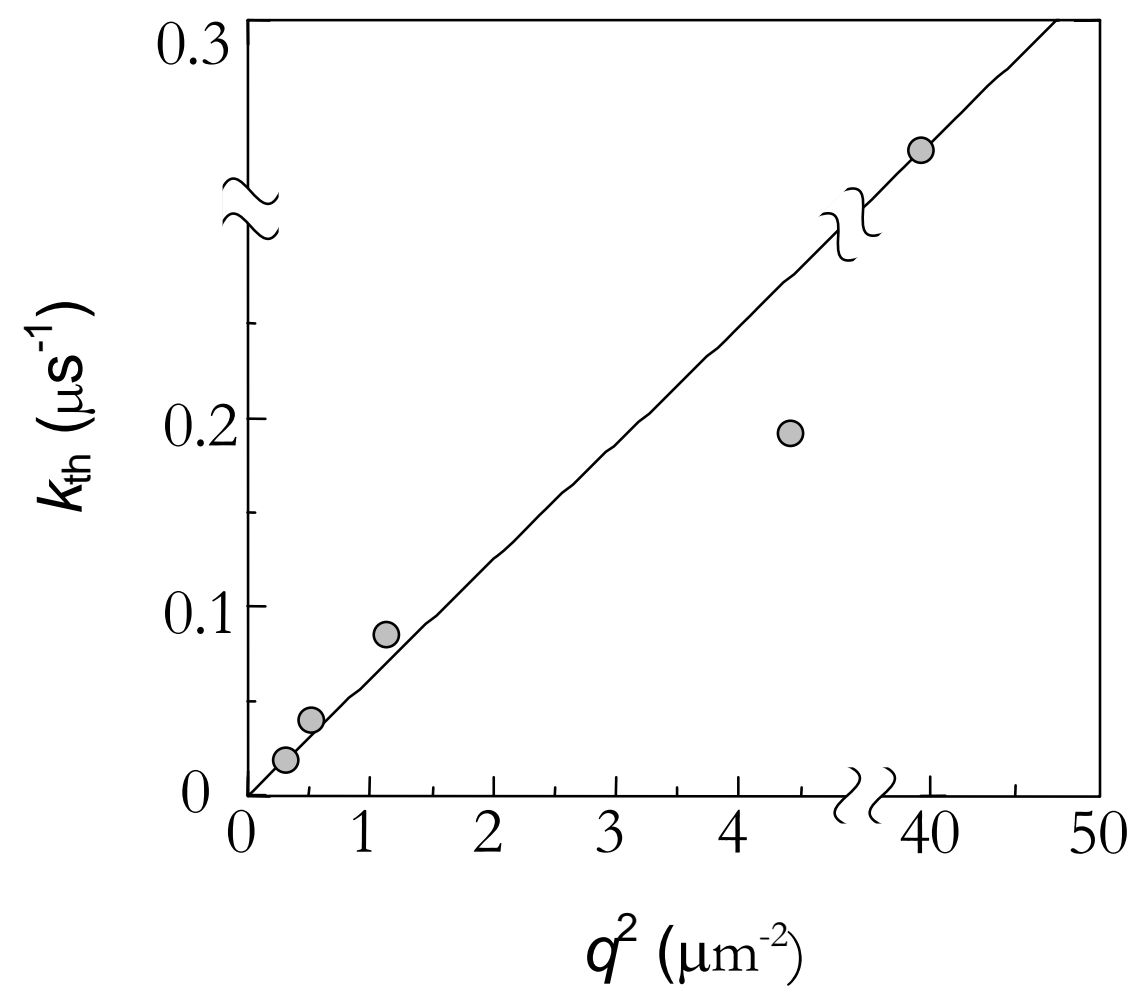

Figure 8.4 Relationship between the square of the grating constants $\left(q^{2}\right)$ and the decay rate $\left(k_{\text {th }}\right)$ of the TG signals. This slope indicates the thermal diffusion coefficient.

$\delta n(t) . q$ is the grating constant described by the grating period $(\Lambda)$ as $q=2 \pi / \Lambda . D_{\text {th }}$ is the thermal diffusion coefficient of solution. Therefore, signal decay rate $\left(k_{\mathrm{th}}=1 / \tau\right)$ obtained by the exponential fitting was described by $k_{\mathrm{th}}=D_{\mathrm{th}} q^{2}$. Figure 8.4 shows the relationship between $k_{\text {th }}$ and $q^{2}$ at each grating period, where a good linear relationship and $D_{\text {th }}$ value is shown by the slope. The obtained value $\left(D_{\text {th }}=7.0 \pm 0.7 \times 10^{8} \mathrm{~m}^{2} \mathrm{~s}^{-1}\right)$ is very close to the calculated value $\left(D_{\text {th }}=6.8 \times 10^{8} \mathrm{~m}^{2} \mathrm{~s}^{-1}\right)[18]$ by the thermal conductivity $(\kappa)$ of 2-propanol as $D_{\text {th }}=\kappa / \rho C_{\mathrm{p}}$. This agreement demonstrates the validity of this method according to signal analysis above. By the same token, several diffusion processes, such as the molecular 
diffusion in solution, energy migration in materials, or the carrier diffusion in semiconductors, should be measurable.

The MPT-TG technique should have many merits for photonic and photochemical application. The experimental setup and the beam alignment of the MPT-TG are simpler and easier than those of the usual OHD-TG technique, while it has very high signal sensitivities and $\mathrm{S} / \mathrm{N}$ ratios for the optical heterodyne detection. The pump and probe beam should be incident at the same spot on the metal grating film, but the tunings of direction and phase of the beams are not necessary. The lens-free OHD-TG technique $[17,18]$ has similar merits. But the MPT-TG technique has still more merits compared to the lens-free technique. In the lens-free technique, an optical interference pattern was constructed by the diffraction light with transmission grating on the sample located behind the grating. The phase differential $\Delta \phi$ can be controlled by the distance $(\sim 250 \mu \mathrm{m}$ usually) between the grating and the sample. In the MPT-TG technique, metal film grating is suspended into the sample solution and the transient grating structure is created directly behind it. Thus, $\Delta \phi$ is decided only by the thickness of the metal layer and the incident angle. Therefore, $\Delta \phi$ stability is controllable by the thickness of the metal layer. Moreover, various patterns can be used flexibly with any metal widths and periods to optimize the sensitivity. The measurement of the grating periods dependence is also very easy to control by sliding the film to change the pattern structure.

\subsection{Conclusion}

In conclusion, our new technique by using nano or micro metal grating film has many advantages compared to the traditional techniques. This technique should be a powerful 
and useful tool for wider applications in physics, chemistry, material, and biological applications.

Bibliography

1. Shen, Y.R., The principles of nonlinear optics. 1984, New York: J. Wiley. xii, 563 p.

2. Eichler, H.J., P. Günter, and D.W. Pohl, Laser-induced dynamic gratings. 1986, Berlin ; New York: Springer-Verlag. xi, 256 p.

3. Fayer, M.D., Dynamics of Molecules in Condensed Phases - Picosecond Holographic Grating Experiments. Annual Review of Physical Chemistry, 1982. 33: p. 63-87.

4. Terazima, M., K. Okamoto, and N. Hirota, Transient radical diffusion in photoinduced bydrogen abstraction reactions of benzophenone probed by the transient grating method. Journal of Physical Chemistry, 1993. 97(50): p. 13387-13393.

5. Dhar, L., J.A. Rogers, and K.A. Nelson, Time-resolved vibrational spectroscopy in the impulsive limit. Chemical Reviews, 1994. 94(1): p. 157-193.

6. Rogers, J.A. and K.A. Nelson, Study of lamb acoustic wave-guide modes in unsupported polyimide thin-films using real-time impulsive stimulated thermal scattering. Journal of Applied Physics, 1994. 75(3): p. 1534-1556.

7. Terazima, M., Translational diffusion of intermediate species in solutions. Research on Chemical Intermediates, 1997. 23(9): p. 853-901.

8. Terazima, M., K. Hirai, and H. Tomioka, Translational diffusion of chemically active species: carbenes and carbonyl oxides. Chemical Physics Letters, 1998. 289(3-4): p. 253-260.

9. Goodno, G.D., G. Dadusc, and R.J.D. Miller, Ultrafast heterodyne-detected transient-grating spectroscopy using diffractive optics. Journal of the Optical Society of America B-Optical Physics, 1998. 15(6): p. 1791-1794. 
10. Goodno, G.D. and R.J.D. Miller, Femtosecond heterodyne-detected four-wave-mixing studies of deterministic protein motions. II. Theory and experimental technique of diffractive optics-based spectroscopy. Journal of Physical Chemistry A, 1999. 103(49): p. 10619-10629.

11. Terazima, M., Optical heterodyne detected transient grating for studies of photochemical reactions and solution dynamics. Chemical Physics Letters, 1999. 304(5-6): p. 343-349.

12. Terazima, M., Optical heterodyne detected transient grating for the separations of phase and amplitude gratings and of different chemical species. Journal of Physical Chemistry A, 1999. 103(37): p. 7401-7407.

13. Dadusc, G., et al., Diffractive optics-based heterodyne-detected four-wave mixing signals of protein motion: From "protein quakes" to ligand escape for myoglobin. Proceedings of the National Academy of Sciences of the United States of America, 2001. 98(11): p. 6110-6115.

14. Torre, R., A. Taschin, and M. Sampoli, Acoustic and relaxation processes in supercooled orthoterphenyl by optical-heterodyne transient grating experiment. Physical Review E, 2001. 6406(6).

15. $\mathrm{Xu}, \mathrm{Q} . \mathrm{H}$., et al., Wavelength-dependent resonant homodyne and heterodyne transient grating spectroscopy with a diffractive optics method: Solvent effect on the third-order signal. Journal of Chemical Physics, 2002. 116(21): p. 9333-9340.

16. Gedik, N., et al., Diffusion of nonequilibrium quasi-particles in a cuprate superconductor. Science, 2003. 300(5624): p. 1410-1412.

17. Katayama, K., M. Yamaguchi, and T. Sawada, Lens-free heterodyne detection for transient grating experiments. Applied Physics Letters, 2003. 82(17): p. 2775-2777.

18. Yamaguchi, M., K. Katayama, and T. Sawada, Lens-free heterodyne transient grating method for dynamics measurement of photoexcited species in liquid. Chemical Physics Letters, 2003. 377(5-6): p. 589-594.

19. Yip, R.W., et al., Picosecond excited-state absorption of alkyl nitrobenzenes in solution. Journal of Physical Chemistry, 1984. 88(24): p. 5770-5772.

20. Terazima, M., K. Okamoto, and N. Hirota, Diffusion process of methyl red in organic-solvents studied by the transient grating method. Journal of Physical Chemistry, 1993. 97(19): p. 51885192. 